\title{
Computer simulations of two-dimensional melting with dipole-dipole interactions
}

\author{
S.Z. Lin $^{1}$, B. Zheng ${ }^{1,2}$ and S. Trimper ${ }^{2}$ \\ 1 Zhejiang University, \\ Zhejiang Institute of Modern Physics, \\ Hangzhou 310027, P.R. China \\ 2 FB Physik, Universität - Halle, \\ 06099 Halle, Germany
}

\begin{abstract}
We perform molecular dynamics and Monte Carlo simulations of two-dimensional melting with dipole-dipole interactions. Both static and dynamic behaviors are examined. In the isotropic liquid phase, the bond orientational correlation length $\xi_{6}$ and susceptibility $\chi_{6}$ are measured, and the data are fitted to the theoretical ansatz. An algebraic decay is detected for both spatial and temporal bond orientational correlation functions in an intermediate temperature regime, and it provides an explicit evidence for the existence of the hexatic phase. From the finite-size scaling analysis of the global bond orientational order parameter, the disclination unbinding temperature $T_{i}$ is estimated. In addition, from dynamic Monte Carlo simulations of the positional order parameter, we extract the critical exponents at the dislocation unbinding temperature $T_{m}$. All the results are in agreement with those from experiments and support the KTHNY theory.

PACS numbers: $64.70 . \mathrm{Dv}, 64.60 . \mathrm{Ht}$
\end{abstract}




\section{INTRODUCTION}

Two-dimensional melting has been intensively studied in the past years, but it is still not completely understood [1, 2, 3]. Melting in two dimensions is quite different from its counterpart in three dimensions, for a true long-range positional order doesn't exist in two-dimensional systems. The absence of a conventional long-range order at non-zero temperature was first pointed out by Mermin and Wagner [4]. Nevertheless, another longrange order, which is called the bond orientational order, can be observed in the solid phase [5].

There exist several possible theoretical descriptions of melting in two-dimensional systems. The KTHNY theory, developed by Halperin, Nelson and Young [ㅁ, 7, 8], predicts that a third phase, the so-called hexatic phase, may exist between solid and liquid states in a portion of the phase diagram. The system first melts from the solid state to the hexatic state due to the unbinding of dislocation at a temperature $T_{m}$, and then melts from the hexatic state to the liquid state at the disclination unbinding temperature $T_{i}$. Both transitions are Kosterlitz-Thouless phase transitions [9]. Naturally, the KTHNY theory only describes a possible scenario. It is also possible that anyone or both of the continuous transitions are of first order, and even that there is a direct first-order transition from the solid state to the isotropic liquid state.

Even though quite some experiments supported the KTHNY theory [10, 11, 12, 13, 14, 15, 16, 17], most early works of computer simulations on two-dimensional melting favored a first-order phase transition, and the hexatic phase was not observed. For example, for the systems with dipole-dipole interactions, Kalia and Vashishta [18] observed a superheating and supercooling, as well as a latent heat in two-dimensional melting, and concluded that the phase transition is of first order. Later, Bedanov, Gadiyak and Lozovik [19] found that both the positional and bond orientational order vanished simultaneously at the melting point, and the hexatic phase didn't exist. Similar results have been found for other systems [20, 21, 22, 23]. Even for the simplest system, the hard disk model, there was no consensus about the melting mechanism [23, 24, 25, 26, 27].

In 1996, Bagchi et al. [28] carried out a finite-size scaling analysis of the bond orientational order parameter in a system interacting via a repulsive $1 / r^{12}$ potential, and found that the results were in agreement with the KTHNY theory, even though no conclusive evidence 
for the hexatic phase was observed. Later, extensive Monte Carlo simulations of the hard disk model were performed by Jaster [29, 30]. Numerical behaviors of the susceptibility, spatial bond orientational correlation length and pressure, support the KTHNY theory. But the algebraic decay of the bond orientational correlation function was still not shown [31]. Recently, Monte Carlo simulations of a two-dimensional electron system with a $1 / r$ interacting potential have been performed by He et al. [32]. An algebraic decay of the bond orientational correlation function is observed, and it explicitly reveals the existence of the hexatic phase. In principle, however, the finite-size effect and coexistence of liquid and solid phases may also lead to such an algebraic decay. One needs to carefully rule out these possibilities. On the other hand, in all these numerical simulations of the bond orientational order, the static behavior of the melting is mainly concerned, and the dynamics is not touched so much.

Recently, more progress in computer simulations has been made in understanding twodimensional melting, for example, on the roles of the polydispersity [33, 34] and external fields [35], and on the structural change during the melting [36]. Especially, some experiments show much interest in a two-dimensional system with dipole-dipole interactions [17, 37]. The algebraic decay of the spatial and temporal correlation functions are observed and the dynamic behavior is found to be very relevant for two-dimensional melting. From the view of numerical simulations, the two-dimensional system with dipole-dipole interactions is not much understood. The purpose of this article is to present systematic computer simulations of two-dimensional melting with dipole-dipole interactions. Main results are obtained with molecular dynamics simulations, and Monte Carlo simulations are also performed in some cases and for confirmation. Both static and dynamic behavior will be examined, and an emphasis is given to the algebraic decay of both the spatial and temporal bond orientational correlation functions in the hexatic phase.

The article is organized as follows. In Sec. II, the model and numerical methods will be described. In Sec. III, numerical results will be presented for both static and dynamic behavior. Finally it comes the conclusion. 


\section{MODEL AND METHOD}

\section{A. The model}

In this article, we consider a two-dimensional dipolar system whose Hamiltonian can be written as

$$
H=\sum_{i}^{N} \frac{p_{i}^{2}}{\mu_{i}}+\frac{1}{2} \sum_{i=1}^{N} \sum_{j \neq i}^{N}\left\{\frac{\overrightarrow{m_{i}} \cdot \overrightarrow{m_{j}}}{\left|\overrightarrow{r_{i j}}\right|^{3}}-3 \frac{\left(\overrightarrow{m_{i}} \cdot \overrightarrow{r_{i j}}\right)\left(\overrightarrow{m_{j}} \cdot \overrightarrow{r_{i j}}\right)}{\left|\overrightarrow{r_{i j}}\right|^{5}}\right\},
$$

where $p_{i}, \mu_{i}$ and $\vec{m}_{i}$ are the momentum, mass and magnetization of the $i$ th dipole respectively, and $N$ is the total number of particles. In order to mimic the experiments in Refs. [17, 37] and to simplify the problem, we assume the dipoles are aligned perpendicular to the surface. Thus, Eq. (1) can be reduced to

$$
H=\sum_{i}^{N} \frac{p_{i}^{2}}{\mu_{i}}+\frac{1}{2} \sum_{i=1}^{N} \sum_{j \neq i}^{N} \frac{m_{i} m_{j}}{\left|\vec{r}_{i j}\right|^{3}} .
$$

For convenience in numerical simulations, we rewrite Eq. (2) as

$$
H=\sum_{i}^{N} \frac{p_{i}^{2}}{\mu}+\frac{1}{2} \sum_{i=1}^{N} \sum_{j \neq i}^{N} \epsilon\left(\frac{\sigma}{r_{i j}}\right)^{3}
$$

where we have assumed the mass and magnitude of the magnetization of the dipoles are identical. For simplification, the reduced units are adopted, in which the parameters $\epsilon$ and $\sigma$, Boltzmann constant $k_{B}$, and mass $\mu$ of the dipoles are set to 1 . The thermodynamic observables are determined only by a dimensionless constant $\Gamma=\epsilon \sigma^{3}(\pi n)^{3 / 2} / k T$ [38] , where $n=N / V$ is the $2 D$ volume fraction of the dipoles.

The reasons we choose this model are: (i) this model lacks extensive numerical study, and the existing results do not favor the KTHNY theory; (ii) there are unambiguous experimental results of such system [17, 37], to which we may compare our results.

\section{B. Numerical methods}

In our simulations, particles are put in a rectangular box with a size ratio $2: \sqrt{3}$, the density of the particles is fixed to be $1 /(2 \sqrt{3})$, and the number of the particles is taken to be from 1024 to 32768 . The linear size $L$ of the system is related to the total number $N$ of particles by $L=2 \sqrt{N}$. Periodic boundary conditions are used in simulations, and the dipole-dipole potential is truncated at 10. In two dimensions, such a truncation is reasonable. 
Actually, the correction of the potential to the truncation is $u_{c}=\int_{0}^{2 \pi} \int_{r_{c}}^{+\infty} g(r) \epsilon\left(\frac{\sigma}{r}\right)^{3} r d r d \theta$; assuming $g(r)=1$, it leads to $u_{c}=2 \pi \epsilon \sigma^{3} / r_{c}$, which decays to zero with $r_{c}$. In fact, the main parts of the simulations are carried out in the hexatic and liquid phases where $g(r)$ quickly

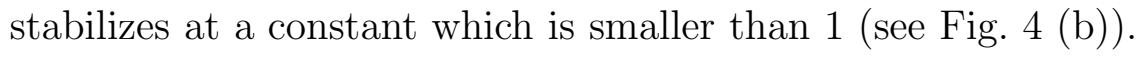

In order to confirm the truncating procedure, we have performed the simulations at different truncating distances, and find that the difference is negligibly small. In addition, extra simulations using the Ewald Summation technique[39, 40], which is known for transferring long-range interactions to short-range ones, are also performed to further justify our truncation. Within statistical errors, the results for the global bond orientational order parameter $\Psi_{6}$ and susceptibility $\chi_{6}$ obtained with different truncating distances and the Ewald Summation are in good agreement with each other. Relevant data with $L=64$ are compiled in table \for comparison. Additional simulations with $L=128$, and measurements of the pair distribution function $g(r)$ also confirm the reliability of the truncation.

In this paper, most simulations are performed with molecular dynamics. All results are obtained at a constant temperature with the $N V T$ ensemble based on the Nosé-Hoover Chain thermostat [41, 42]. The equation of the motion is solved via the five-point NordsieckGear predictor-corrector method. The time step $\Delta t$ in all the simulations is set to 0.01 . A shift of the conserved total energy is within $0.0001 \%$.

The initial configurations in our simulations consist of particles uniformly distributed over the system box on a triangular lattice. Before collecting data for the measurements of physical observables, the system is carefully equilibrated, especially in the critical regime. We monitor the global bond orientational order parameter, and begin our measurement after this order parameter reaches a steady value. For the larger system $(N=16384)$, for example, it takes $5 \times 10^{5}$ time steps to thermalize the system. Only the configurations in equilibrium are used for the measurements of observables, extending over $9 \times 10^{6}$ time steps. In order to obtain independent configurations, the autocorrelation function of the global bond orientational order parameter is measured, and the correlation time is estimated to be $\tau \approx 2400$ time steps in the critical regime. Then the measurement is performed every 2500 time steps.

In order to confirm our molecular dynamics simulations, standard Monte Carlo simulations are additionally performed. For example, The bond orientational correlation functions from both molecular dynamics simulations and Monte Carlo simulations are shown in 
Fig. 1 (a). Both methods provide consistent curves, and it shows that our molecular dynamics simulations indeed generate proper ensemble distributions. Furthermore, dynamic Monte Carlo simulations are carried out to extract the critical exponents for the positional order parameter at the dislocation unbinding temperature $T_{m}$.

\section{Observables}

The bond orientational symmetry of a solid can be described by the six-fold global bond orientational order parameter $\Psi_{6}$ defined as

$$
\Psi_{6}=\left\langle\left|\frac{1}{N} \sum_{k=1}^{N} \psi_{6, k}\right|\right\rangle
$$

where $N$ is the total number of the particles, $\langle\cdots\rangle$ denotes the ensemble average or the time average in molecular dynamics simulations and Monte Carlo simulations, and the $\psi_{6, k}$ is the local bond orientational order parameter defined as

$$
\psi_{6, k}=\frac{1}{N_{k}} \sum_{j} \exp \left(i 6 \theta_{k j}\right) .
$$

Here the sum $\mathrm{j}$ is over the neighbors of the particle $\mathrm{k}$, and $\theta_{k j}$ is the angle between $\overrightarrow{r_{k j}}$ (the relative position vector of the particle $k$ and $j$ ) and an arbitrarily fixed reference axis. Neighbors are obtained with the Voronoi polygon [43]. The susceptibility of the bond orientational order is defined as

$$
\chi_{6}=N\left\langle\Psi_{6}^{2}\right\rangle .
$$

The hexatic phase is characterized by an algebraic decay of the bond orientational correlation function defined as

$$
g_{6}\left(\overrightarrow{r_{1}}-\overrightarrow{r_{2}}\right)=\left\langle\psi_{6, k}^{*}\left(\overrightarrow{r_{1}}\right) \psi_{6, k}\left(\overrightarrow{r_{2}}\right)\right\rangle
$$

In order to obtain an accurate value of the bond correlation length, we smooth the bond orientational correlation function following Ref. [30]. We divide the volume of the system into stripes with a width of $\Delta x$ and measure the bond orientational correlation between different stripes.

$$
g_{6}(x)=\left\langle\left(\frac{1}{N(x)} \int_{0}^{L} \mathrm{~d} y^{\prime} \int_{x-\Delta x / 2}^{x+\Delta x / 2} \mathrm{~d} x^{\prime} \psi_{6, k}\left(\overrightarrow{r^{\prime}}\right)\right)^{*} \times\left(\frac{1}{N(0)} \int_{0}^{L} \mathrm{~d} y^{\prime} \int_{-\Delta x / 2}^{\Delta x / 2} \mathrm{~d} x^{\prime} \psi_{6, k}\left(\overrightarrow{r^{\prime}}\right)\right)\right\rangle,
$$


where

$$
\begin{gathered}
N(x)=\int_{0}^{L} \mathrm{~d} y^{\prime} \int_{x-\Delta x / 2}^{x+\Delta x / 2} \mathrm{~d} x^{\prime} \rho\left(\overrightarrow{r^{\prime}}\right), \\
\rho(\vec{r})=\sum_{i=1}^{N} \delta\left(\vec{r}-\overrightarrow{r_{i}}\right),
\end{gathered}
$$

and $L$ is the linear size of the system in the $y$ direction. The temporal bond orientational correlation function characterizes the time correlation of the bond orientational order parameter, and is defined as

$$
g_{6}(t)=\left\langle\psi_{6, k}^{*}\left(t_{0}\right) \psi_{6, k}\left(t_{0}+t\right)\right\rangle,
$$

where $\psi_{6, k}\left(t_{0}\right)$ and $\psi_{6, k}\left(t_{0}+t\right)$ are the local bond orientational order parameters measure at the time $t_{0}$ and $t_{0}+t$ respectively, and the average is over $t_{0}$ in equilibrium. In the hexatic phase, $g_{6}(t)$ also decays by a power law [37].

The positional symmetry of solid can be described by a positional order parameter defined as

$$
\Psi_{\text {pos }}=\left\langle\frac{1}{N} \sum_{j=1}^{N} \exp \left(i \vec{G} \cdot \vec{r}_{j}\right)\right\rangle,
$$

where $G$ is a reciprocal-lattice vector which gives the first Bragg peak. In practice, we average the order parameter over the six reciprocal vectors which correspond to the six vectors connecting the six neighbors from the lattice site $j$. The positional correlation function is defined as

$$
g_{G}\left(\left|\vec{r}-\overrightarrow{r^{\prime}}\right|\right)=\left\langle\exp \left(i \vec{G} \cdot\left(\vec{r}-\overrightarrow{r^{\prime}}\right)\right)\right\rangle
$$

In the hexatic phase, the positional correlation function decays exponentially. Finally, the pair distribution function is defined as

$$
g(r)=\frac{V}{N^{2}} \sum_{i, j \neq i} \delta\left(\vec{r}-\vec{r}_{i j}\right),
$$

where $V$ is the volume of the system.

\section{COMPUTER SIMULATION}

In this article, we perform extensive simulations of two-dimensional melting in the $N V T$ ensemble with system sizes up to 32786 atoms, and find a strong evidence for the existence of the hexatic phase in the dipole-dipole interacting system. We first measure the spatial bond orientational correlation function and susceptibility in the isotropic liquid phase and 
compare the results with the predictions of the KTHNY theory. This gives us estimates of the isotropic-anisotropic transition temperature $T_{i}$. With this critical temperature in hand, we further scan the parameter space, and observe an algebraic decay of the spatial bond orientational correlation. We also measure the temporal bond orientational correlation function, and its behavior is in good agreement with the KTHNY theory. In order to rule out a possible coexistence phase and the finite-size effect, we perform a homogeneous test and finite-size scaling analysis of the bond orientational order parameter. The result is compatible with previous measurements. At last, with Monte Carlo methods we simulate the short-time dynamics of the positional order and estimate the exponent $\eta_{m}$, and the value is also in agreement with the theoretical prediction. All our results are compatible with the experiments and KTHNY theory, and the hexatic phase is explicitly observed.

\section{A. Bond orientational order}

The bond orientational order parameter $\Psi_{6}$ offers a direct description of the bond orientational order [23]. Assuming $T_{i}$ is the transition temperature of the bond orientational order and $T_{m}$ is the transition temperature of the positional order, the bond orientational order parameter should vanish for $T>T_{i}$. and take a finite value less than 1 for $T<T_{m}$. The behavior of $\Psi_{6}$ at the temperatures between $T_{i}$ and $T_{m}$ depends on the underlying melting scenario. If the transition at $T_{i}$ is of first order, $\Psi_{6}$ increases linearly from $T_{i}$ to $T_{m}$. If the melting scenario is of KTHNY, i.e., the transition at $T_{i}$ is a Kosterlitz-Thouless phase transtion, $\Psi_{6}$ then vanishes throughout the hexatic phase for there doesn't exist a true longrange bond orientational order. However, the finite-size effect in the simulations blurs this distinction and prevents us drawing a clear conclusion. Nevertheless, the measurement of the bond orientational order parameter $\Psi_{6}$ does give us an estimated value of $T_{i} \approx 0.01250$. In the Fig. 1 (b), $\Psi_{6}$ versus $T$ is shown.

To further understand the phase transition at $T_{i}$, we measure the correlation length and susceptibility of the bond orientational order parameter in the isotropic liquid phase for different temperature $T$. For the measurements are carried out in the isotropic liquid phase, the spatial bond orientational correlation function is independent of the spatial directions. We extract the correlation length $\xi$ from the exponential decay of the bond orientational 
correlation function smoothed with the technique described in Eq. (88),

$$
g_{6}(x) \sim \exp (-x / \xi)
$$

Subsequently, we compare our results with the predictions of the KTHNY theory, i.e., an exponential singularity of the correlation length and susceptibility,

$$
\begin{gathered}
\xi_{6}(\tau)=a_{\xi} \exp \left(b_{\xi} \tau^{-1 / 2}\right), \\
\chi_{6}(\tau)=a_{\chi} \exp \left(b_{\chi} \tau^{-1 / 2}\right),
\end{gathered}
$$

as $\tau=T-T_{i} \rightarrow 0^{+}$. In Fig. 2, the numerical data are fitted to the above exponential forms. The best fit of the correlation length and susceptibility gives $T_{i}=0.01237(16)$ and $T_{i}=0.01243(4)$ respectively. These two values are in agreement with each other within statistical errors, and are also consistent with the previous estimated value of $T_{i}$ from the global bond orientational order parameter. Therefore, our results support the KTHNY

prediction, even though the statistical errors of the correlation length and susceptibility are relatively large.

\section{B. The hexatic phase}

According to the KTHNY theory, the hexatic phase is characterized by an algebraic decay of the bond orientational correlation function and an exponential decay of the positional correlation function. Therefore, we scan the parameter space between $T_{i}$ and $T_{m}$, and measure the bond orientational correlation function and positional correlation function. The bond orientational correlation function is shown in Fig. 3 (a). A clear evidence for the existence of the hexatic phase is observed.

i) In the solid phase $(T=0.0119)$, the correlation function rapidly stabilizes to a constant, and it indicates that there is a true long-range order of the bond orientational symmetry.

ii) In the hexatic phase $(T=0.01252$ and 0.01253$)$, the correlation function shows an algebraic decay,

$$
g_{6}(x) \sim x^{-\eta_{6}},
$$

and it indicates that there is a quasi-long-range order of the bond orientational symmetry.

iii) In the liquid phase $(T=0.0127)$, the correlation function decays exponentially, and it indicates an isotropic state. 
After smoothing the correlation function at $T=0.01253$, we obtain an exponent $\eta_{6}=$ 0.252 (6) from the slope of the curve, and it is close to the value $\eta_{6}=0.25$ at the transition temperature $T_{i}$ predicted by the KTHNY theory. If we assume $T=0.01253$ is just the transition temperature $T_{i}$, it is quantitatively in agreement with the previous measurements in the preceding subsection. Nevertheless, to obtain a more accurate value of $T_{i}$, we need to consider more carefully the finite-size effect. In the next subsection, we will locate the transition temperature from the finite-size scaling.

In Fig. 4, the positional correlation function and pair distribution function are shown respectively. One may observe two different behaviors.

i) In the solid phase $\left(T_{i}=0.0119\right)$, the positional correlation function shows an algebraic decay, indicating a quasi-long-range positional order in a two-dimensional solid, while the oscillation in the pair distribution function persists over the entire range.

ii) In the hexatic phase $\left(T_{i}=0.01253\right)$, the positional correlation function decays quickly to zero, indicating that there exists no positional order in the hexatic phase, and the oscillation in the pair distribution function dies off rapidly. The behaviors of the positional correlation function and pair distribution function in the liquid phase are qualitatively the same as in the hexatic phase.

The recent experiment reported in Ref. [37] shows that the dynamic behavior is also very important in understanding the melting mechanism in two dimensions. According to the KTHNY theory, in the solid phase the temporal bond orientation correlation function will rapidly stabilize to a constant, in the hexatic phase it shows an algebraic decay with an exponent equal to $\eta_{6} / 2$,

$$
g_{6}(t) \sim t^{-\eta_{6} / 2},
$$

and in the liquid phase the temporal correlation function decays exponentially [44]. Such a behavior is indeed observed in experiments, and it provides a strong evidence for the existence of the hexatic phase. To our knowledge, such measurements have not been performed in numerical simulations.

In order to deepen our understanding of two-dimensional melting and further confirm our observation in numerical simulations of static properties, the temporal bond orientation correlation function is measured in our molecular dynamics simulations. The result is shown in Fig. 3(b). Obviously, as the temperature changes from $T=0.0119$ to 0.01253 , then to 0.0131 , the temporal bond orientation correlation function follows the prediction of 
the KTHNY theory, and are well consistent with the experimental observation [37]. The exponent measured from the slope of the curve at $T=0.01253$ is 0.0843 , somewhat smaller than the theoretical prediction 0.125 at $T_{i}$. This probably suggests that the anisotropicisotropic transition temperature $T_{i}$ should be still slightly above the value 0.01253 , and our measurements of the spatial and temporal bond orientational correlation functions may still carry certain finite-size effects.

\section{Finite-size scaling analysis}

Our measurements of the spatial and temporal bond correlation functions provide us an explicit evidence for the existence of the hexatic phase in two-dimensional melting. However, it is difficult to extract an accurate transition temperature $T_{i}$ from the correlations functions. One may directly measure the correlation length in the isotropic liquid phase and then fit the data to the ansatz in Eq. (16) and obtain the transition temperature $T_{i}$. But this approach also suffers from the difficulty that one can not obtain the correlation length at the temperatures very close to $T_{i}$ [45, 46]. Meanwhile, due to the finite-size effect, distinguishing between an algebraic and an exponential decay might be problematic if the correlation length is finite but much larger than the system size. Therefore, to extract a more accurate disclination unbinding temperature $T_{i}$ and to confirm the previous observation of the hexatic phase, we perform a finite-size scaling analysis of the bond orientational order parameter.

From the finite-size scaling form, the second moment of the bond orientational order parameter can be written as

$$
\left\langle\Psi_{6}^{2}\right\rangle \sim L^{-\eta_{6}} f\left(L / \xi_{6}\right)
$$

where $L=2 \sqrt{N}$ is the linear size of the system and $\xi_{6}$ is the bond correlation length. Since $\xi_{6}$ is divergent in the hexatic phase, $\left\langle\Psi_{6}^{2}\right\rangle$ thus shows a power-law behavior with respect to $L$ in the hexatic phase. In the liquid phase, the power-law behavior will be modified by the scaling function $f\left(L / \xi_{6}\right)$.

We measure the second moment of global bond orientational order parameter with system size $L=64,128,256$ at $T=0.01257$ and perform finite-size scaling analysis mentioned above. The open circles shown in Fig. 5 are the results. In order to save computation time, we use the subsystem method introduced by the authors of Refs. [23, 28]. Here, a brief 
comment about the above non-standard finite-size scaling analysis is needed. In principle, the subsystem procedure still carries a second-order finite-size effect induced by the finite bulk system size $L$. But this second-order finite-size effect is negligibly small in practical simulations [23], and the procedure has been proved to be reliable and may reduce computer times [28]. We also carried out the finite-size scaling analysis using subsystem method at $T=0.01257$ to further justify this procedure, the result is shown in Fig. 5. It is easy to observe that within statistical errors, the data with periodic boundary conditions and from subsystems are well consistent. With the subsystem method, we measure $\left\langle\Psi_{6}^{2}\right\rangle$ at different temperatures with a bulk linear size $L=256$ or 512 , and a total number of particles ranging from $N=16384$ to 32768 . Then the system is divided into small subsystems with a linear size $L_{S}$ [23] and the bond orientational order parameter of each subsystem is measured. The result is shown in Fig. 5 .

To locate $T_{i}$, we assume $\eta_{6}=1 / 4$. In other words, we search for a temperature which yields $\eta_{6}=1 / 4$, and then assign this temperature to be $T_{i}$. The requirement of $\eta_{6}=1 / 4$ yields the upper limit of $T_{i}[44]$. Combining the results obtained in the preceding subsections, we conclude $0.01253<T_{i}<0.01257$. To compare our results with those in literatures, we convert $T_{i}$ to the dimensionless parameter $\Gamma_{i}$, and obtain $68.707<\Gamma_{i}<68.927$. It improves the values $T_{i}=62 \pm 3$ with a small system $N=256$ [18] and $T_{i}=67.750$ with a relatively larger system $N=961$ [47]. In Refs.[18, 47], the phase transition is supposed to be of first order, and the values of $T_{i}$ are obtained from the hysteresis in the temperature dependence of energy, the existence of latent heat and the thermodynamic nucleation of the solid from the supercooled liquid. Our estimate of $T_{i}$ is based on the KTHNY theory, and is much less affected by the finite-size effect.

\section{Ruling out the coexistence phase}

In principle, the $N V T$ molecular dynamics simulation can not obviate the coexistence phase, and the superposition of the solid and liquid phases may also produce the hexatic-like behavior. In order to exclude this possibility, we apply the homogeneous test. We divide the system into small subsystems and compute the susceptibility $\chi_{6}$ for all subsystems [21]. If the system exhibits an inhomogeneous two-phase coexistence, the probability distribution of $\chi_{6}$ at a sufficiently small length scale could be modeled by a curve with two peaks, which 
reflects a combination of solid and fluid distributions. On the other hand, if the system is homogeneous, varying the size of the subsystems should not lead to any qualitative change in the probability distribution of $\chi_{6}$, i.e., the curve should always remain with a single peak.

We have measured the possibility distribution of $\chi_{6}$ in the hexatic phase at $T=$ 0.01257, 0.01253, 0.01252, and in order to compare the result with that in the homogeneous phase, we also perform a simulation at an extra temperature $T=0.0100$ corresponding to the cool solid phase. No qualitatively change is found at these temperatures. This test rules out the existence of a coexistence phase, and confirms the observation of the hexatic phase in the previous subsections. The result at $T=0.01252$ is shown in Fig. 6. It is clearly seen that varying the system size doesn't change the shape of distributions, but only shifts the peak of the probability distribution.

\section{E. Dynamic Monte Carlo simulations}

In the last decade, it has been discovered that already in a macroscopic short-time regime emerges the universal scaling behavior [48, 49, 50, 51, 52]. Measurements now are carried out at the early stage of the time evolution, therefore one does not suffer from critical showing down. The dynamic scaling form of the second moment of the positional order parameter below the dislocation unbinding transition temperature $T_{m}$ is

$$
\Psi_{\text {pos }}^{2}(t, L)=b^{-\eta_{m}} \Psi_{\text {pos }}^{2}\left(b^{-z} t, b^{-1} L\right)
$$

where $t$ is the evolution time, $z$ is the dynamic critical exponent, and $b$ is an arbitrary rescaling factor. For a sufficient large $L$, this dynamic scaling form is reduced to

$$
\Psi_{p o s}^{2}(t) \sim t^{-\eta_{m} / z}
$$

From a finite-size scaling analysis of the time-dependent Binder cumulant [48]

$$
U_{\text {pos }}(t)=\frac{\Psi_{\text {pos }}^{4}}{\left(\Psi_{\text {pos }}^{2}\right)^{2}}-1
$$

one obtains

$$
U_{p o s}(t) \sim t^{d / z} / L^{d}
$$

where $d$ is the dimension of the system. The dynamic critical exponent $z$ can be estimated from Eq.(24), and with $z$ in hand, the static exponent $\eta_{m}$ can be obtained from Eq. (22). 
Now, we turn to locate the transition temperature $T_{m}$. As the temperature increases, $T_{m}$ is characterized by the dislocation unbinding which breaks the quasi-long-range positional symmetry. Therefore, one may measure the correlation function of the positional order parameter to estimate $T_{m}$, for the positional correlation become short-range at $T_{m}$. Nevertheless, this method suffers from the difficulty that one needs to do simulations in the critical region. Even for the hard disk model, in which the thermodynamic quantities are only determined by the density $\rho$ of disks, it is still not easy to locate $\rho_{m}$ accurately. $\rho_{m} \approx 0.933$ is reported in Ref.[50], while $\rho_{m}=0.910(2)$ is given in Ref. [52].

Alternatively, a dynamic technique for locating $T_{m}$ is applied in the experiments reported in Ref. [37]. In our computer simulations, we follow Ref. [37] and adopt the dynamic criterion for $T_{m}$, since the method is relatively simple, and may provide direct comparison with experiments. We first introduce the $2 D$ Lindemann parameter [17, 53]

$$
\gamma_{m}=\left\langle\left|\vec{u}\left(\vec{r}+\overrightarrow{a_{0}}\right)-\vec{u}(\vec{r})\right|^{2}\right\rangle \times \pi n
$$

where $\overrightarrow{a_{0}}$ is the lattice spacing vector, $\vec{r}$ is the positional vector, $\vec{u}$ is the displacement field and $n$ is the $2 D$ volume fraction of particles. Initially, the system is set on a perfect triangular lattice. In numerical simulations, we gradually warm up the system with the velocity rescaling procedure. At each $T$, the system is equilibrated to the thermal equilibrium. Then we measure the Lindemann parameter at different temperatures. In general, a sharp growth of $\gamma_{m}$ indicates vanishing of the positional symmetry. Such a Lindemann criterion in $3 D$ systems is a well-established and justified procedure for locating the melting temperature $T_{m}$, although it was unclear in two dimensions [4]. In 1985, Bedanov and Gadiyak improved the definition of the Lindemann parameter to the form in Eq. (25) and demonstrated in the simulations of electron and dipole systems that when $\gamma_{m}$ rises up to a critical value 0.12 , the melting takes place [53]. At the melting point $T_{m}$, which is more clearly identified by the sudden drop of the positional correlation length, a sharp growth of $\gamma_{m}$ is induced by the leap of disclination number and self-diffusion constant. Therefore, this local quantity is relevant to the melting. Recently, Zahn et al. applied this criterion to their experiments [17, 37], and the results are in agreement with those from numerical simulations [53]. The Lindemann parameter may provide at least a first estimate of the melting temperature $T_{m}$. Due to its efficiency and simplicity, the Lindemann criterion has been applied to different systems for locating the melting point [54, 55, 56]. 
Here we locate $T_{m}$ with the Lindemann criterion. Four runs are performed in order to estimate $T_{m}$. One of them is shown in Fig. 7. We estimate $T_{m}=0.0120(2)$. The hexatic phase of the dipolar system lies in a range of the phase diagram, between $0.01253<$ $T_{i}<0.01257$ and $T_{m}=0.0120(2)$. This is comparable with that of the hard disk model, $\rho_{i}=0.899(1)$ [30], and $\rho_{m} \approx 0.933$ in Ref. [50] while $\rho_{m}=0.910(2)$ in Ref. [52]. $T_{i}$ and $T_{m}$ may overlap for small systems, and this is one reason why the hexatic phase was not observed in some previous studies.

Now we perform dynamic Monte Carlo simulations at the transition temperature $T_{m}$. The reason we perform Monte Carlo simulations is that the dynamic scaling forms in Eqs. (22) and (24) may not hold in the dynamic process of Nosé-Hoover chain molecular dynamics simulations. It seems that the Nosé-Hoover Chain method is originally devised for equilibrium simulations and contains techniques violating the dynamic scaling behavior. In comparison to this, the dynamic scaling behavior in Monte Carlo simulations has been extensively justified.

In Monte Carlo simulations, the system initially at an ordered state is released to the dynamic evolution with the Metropolis algorithm, and then the time-dependent $\Psi_{\text {pos }}^{2}$ and $U_{\text {pos }}$ are measured. By fitting $\Psi_{\text {pos }}^{2}(t)$ and $U_{\text {pos }}(t)$ to Eqs. (22) and (24), both the dynamic exponent $z$ and static exponent $\eta_{m}$ can be determined. For comparison, we also perform the same simulations at another temperature $T=0.0115$. The results are shown in Fig. 8 (a) and (b).

From $U_{\text {pos }}(t)$ in Fig. 8 (b), we estimate $z=1.910(70)$, and from $\Psi_{\text {pos }}^{2}(t)$ in Fig. 8 (a), we measure $\eta_{m} / z=0.143(5)$. Combining these results, we deduce $\eta_{m}=0.273(20)$. This value is also in agreement with the prediction $\left(1 / 4 \leq \eta_{m} \leq 1 / 3\right)$ based on the KTHNY theory [1].

\section{CONCLUSION}

We present molecular dynamics and Monte Carlo simulations of two-dimensional melting with dipole-dipole interactions. An algebraic decay is observed for both the spatial and temporal bond orientational correlation functions in an intermediate temperature regime, and this serves as an explicit evidence for the existence of the hexatic phase.

To obtain a relatively accurate disclination unbinding temperature $T_{i}$, we perform a finite- 
size scaling analysis for the bond orientational order parameter. The result $0.01253<T_{i}<$ 0.01257 improves the value from a direct fit of the correlation length to the exponential ansatz. In addition, by analyzing the probability distribution of the bond orientational susceptibility $\chi_{6}$, a possible coexistence phase is ruled out.

At last, from dynamic behavior of the Lindemann parameter, the dislocation unbinding transition temperature is estimated to be $T_{m}=0.0120(2)$. We also perform dynamic Monte Carlo simulations of the positional order parameter and the time-dependent cumulant. From the power-law behavior of these quantities, we determine the exponents $\eta_{m}=0.273(20)$ and $z=1.910(70)$.

In summary, a clear evidence for the existence of the hexatic phase is observed for twodimensional melting with dipole-dipole interactions, and all the static and dynamic behaviors of the system are compatible with recent experiments and the KTHNY theory.

Acknowledgements: The authors would like to thank A. Jaster for helpful discussions and suggestions. Numerical Computations have been performed at the Center for Engineering and Scientific Computation, Zhejiang University. This work is supported in part by NNSF and SRFDP (China) and DFG (Germany).

[1] K.J. Strandburg, Rev. Mod. Phys. 60, 161 (1988).

[2] M.A. Glaser and N.A. Clark, Adv. Chem. Phys. 83, 543 (1993).

[3] J.G. Dash, Rev. Mod. Phys. 71, 1737 (1999).

[4] N.D. Mermin and H. Wagner, Phys. Rev. Lett. 17, 1133 (1966).

[5] N.D. Mermin, Phys. Rev. 176, 250 (1968).

[6] B.I. Halperin and D.R. Nelson, Phys. Rev. Lett. 41, 121 (1978).

[7] D.R. Nelson and B.I. Halperin, Phys. Rev. B 19, 2457 (1979).

[8] A.P. Young, Phys. Rev. B 19, 1855 (1979).

[9] J.M. Kosterlitz and D.J. Thouless, J. Phys. C 6, 1181 (1973).

[10] S.C. Davey, J. Budai, J.W. Goodby, R. Pindak, and D.E. Moncton, Phys. Rev. Lett. 53, 2129 (1984).

[11] F. Gallet, G. Deville, A. Valdès, and F.I.B. Williams, Phys. Rev. Lett. 49, 212 (1982).

[12] C.J. Guo, D.B. Mast, R. Mehrotra, Y.-Z. Ruan, M.A. Stan, and A.J. Dahm, Phys. Rev. Lett. 
51, 1461 (1983).

[13] J.P. McTague, J. Als-Nielsen, J. Bohr, and M. Nielsen, Phys. Rev. B 25, 7765 (1982).

[14] M. Nielsen, J. Als-Nielsen, J. Bohr, J.P. McTague, D.E. Moncton, and P.W. Stephens, Phys. Rev. B 35, 1419 (1987).

[15] N. Greiser, G.A. Held, R. Frahm, R.L. Greene, P.M. Horn, and R.M. Suter , Phys. Rev. Lett. 59, 1706 (1987).

[16] R.E. Kusner, J.A. Mann, J. Kerins, and A.J. Dahm, Phys. Rev. Lett. 73, 3113 (1994).

[17] K. Zahn, R. Lenke, and G. Maret, Phys. Rev. Lett. 82, 2721 (1999).

[18] R.K. Kalia and P. Vashishta, J. Phys. C 14, L643 (1981).

[19] V.M. Bedanov, G.V. Gadiyak, and Yu.E. Lozovik, Phys. Lett. 92A, 400 (1982).

[20] S. Toxvaerd, Phys. Rev. A 24, 2735 (1981)

[21] K. J. Strandburg, J. A. Zollweg, and G. V. Chester, Phys. Rev. B 30, 2755 (1984).

[22] P.S. Branício, J.P. Rino, and N. Studart, Phys. Rev. B 64, 193413 (2001).

[23] H. Weber, D. Marx, and K. Binder, Phys. Rev. B 51, 14636 (1995).

[24] J.A. Zollweg, G.V. Chester, and P.W. Leung, Phys. Rev. B 39, 9518 (1989).

[25] J.A. Zollweg, G.V. Chester, Phys. Rev. B 46, R11186 (1992).

[26] J.F. Fernández, J.J. Alonso, and J. Stankiewicz, Phys. Rev. Lett. 75, 3477 (1995)

[27] J.F. Fernández, J.J. Alonso, and J. Stankiewicz, Phys. Rev. E 55, 750 (1997)

[28] K. Bagchi, H.C. Andersen, and W. Swope, Phys. Rev. Lett. 76, 255 (1996).

[29] A. Jaster, Europhys. Lett. 42, 277 (1998).

[30] A. Jaster, Phys. Rev. E 59, 2594 (1999).

[31] Recently, from the finite-size scaling behaivor of the bond orientational order parameter and positional order parameter, one finds a clue for the exisitence of the hexatic phase. See A. Jaster, Phys. Lett. A 330, 120 (2004).

[32] W.J. He, T. Cui, Y.M. Ma, Z.M. Liu, and G.T. Zou, Phys. Rev. B 68, 195104 (2003).

[33] S. Pronk and D. Frenkel, Phys. Rev. E 69, 066123 (2004).

[34] H. Watanabe, S. Yukawa, and N. Ito, Phys. Rev. E 71, 016702 (2005).

[35] W. Strepp, S. Sengupta, and P. Nielaba, Phys. Rev. E 66, 056109 (2002).

[36] F. Moučka and I. Nezbeda, Phys. Rev. Lett. 94, 040601 (2005).

[37] K. Zahn and G. Maret, Phys. Rev. Lett. 85, 3656 (2000).

[38] R. Haghgooie and P. S. Doyle, Phys. Rev. E 72, 011405 (2005). 
[39] P. Ewald, Ann. Phys. Z. 58, 253 (1921).

[40] A. Grzybowski and A. Bródka, Chem. Phys. Lett. 361, 329 (2002).

[41] S. Nóse, J. Chem. Phys. 81, 511 (1984).

[42] W.G. Hoover, Phys. Rev. A 31, 1695 (1985).

[43] D.P. Fraser, M.J. Zuckermann, and O.G. Mouritsen, Phys. Rev. A 42, 3186 (1990).

[44] D. Nelson ,in Phase Transition and Critical Phenomena, edited by C. Domb and J. L. Lebowitz (Academic Press, London, 1983)

[45] R. Gupta and C.F. Baillie, Phys. Rev. B 45, 2883 (1992)

[46] B. Zheng, M. Schulz, and S. Trimper, Phys. Rev. E 59, R1351 (1999)

[47] M. Schmidt and H. Löwen (unpublished).

[48] B. Zheng, Int. J. Mod. Phys. B 12, 1419 (1998), review article

[49] H.J. Luo, L. Schülke, B. Zheng, Phys. Rev. Lett. 81, 180 (1998).

[50] A. Jaster, Phys. Lett. A 258, 59 (1999).

[51] B. Zheng, M. Schulz, and S. Trimper, Phys. Rev. Lett. 82, 1891 (1999)

[52] H. Watanabe, S. Yukawa, Y. Ozeki, and N. Ito, Phys. Rev. E 69, 045103(R) (2004).

[53] V.M. Bedanov and G.V. Gadiyak, Phys. Lett. 109A, 289 (1985).

[54] W. A. Al-Saidi and D. Stroud, Phys. Rev. B 67, 024511 (2003).

[55] S. Tyagi and Y.Y. Goldschmidt, Phys. Rev. B 70, 024501 (2004).

[56] G. Rastelli and S. Ciuchi, Phys. Rev. B 71, 184303 (2005).

\begin{tabular}{l|l|l|l|l}
\hline \hline & $\Psi_{6}(T=0.0150)$ & $\Psi_{6}(T=0.0125)$ & $\chi_{6}(T=0.0150)$ & $\chi_{6}(T=0.0125)$ \\
\hline$r_{t}=10$ & $0.0842(25)$ & $0.684(4)$ & $9.14(49)$ & $479(6)$ \\
\hline$r_{t}=20$ & $0.0849(35)$ & $0.680(4)$ & $9.34(38)$ & $475(5)$ \\
\hline Ewald Summation & $0.0859(37)$ & $0.680(1)$ & $9.50(84)$ & $474(2)$ \\
\hline \hline
\end{tabular}

TABLE I: The global bond orientational order parameter $\Psi_{6}$ and susceptibility $\chi_{6}$ measured by truncating the potential at $r_{t}=10, r_{t}=20$ and with the Ewald Summation to deal with the potential. The linear size is $L=64$, and the temperature is $T=0.0150$ and $T=0.0125$. 


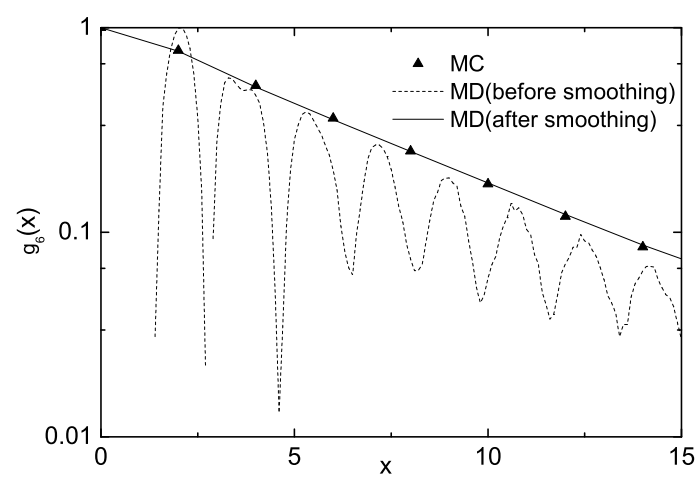

(a)

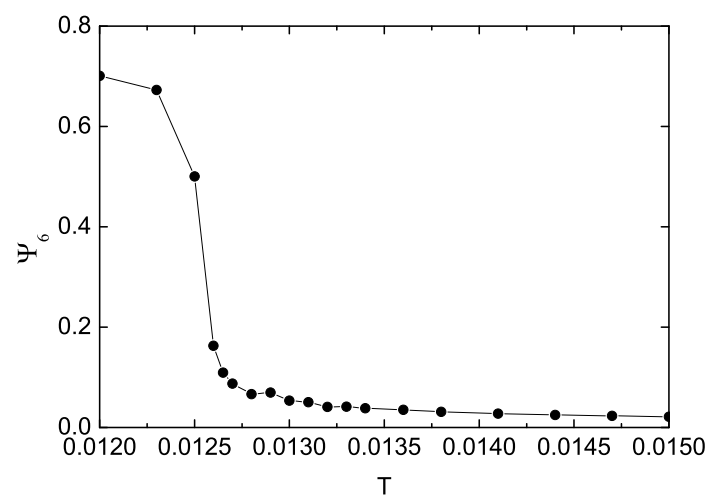

(b)

FIG. 1: (a) $g_{6}(x)$ obtained with molecular dynamics (MD) and Monte Carlo (MC) simulations at $T=0.0150$ plotted vs. $x$ on a semi-log scale. The smoothed curve is shifted upward for clarity. The smoothing technique is described in Sec. II. (b) $\Psi_{6}$ plotted vs. $T$ on a linear plot. The bond orientational order parameter increases abruptly around $T=0.0125$. The line fitted to the circles is a guide to the eyes.

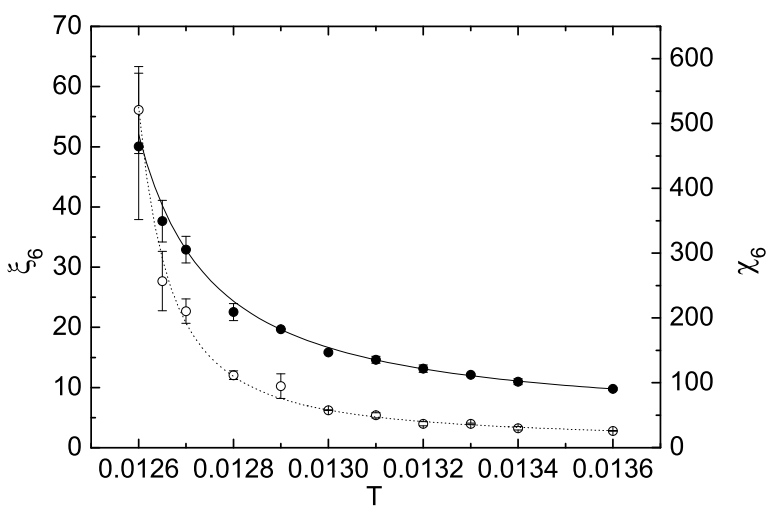

FIG. 2: Bond orientational correlation length (full symbols) and susceptibility (open symbols) as a function of temperature. The curves show the best fits of Eqs. (16) and (17) according to the KTHNY theory. The fitted transition temperatures are $T_{i}=0.01237(16)$ and $0.01243(4)$ for the correlation length and susceptibility respectively 


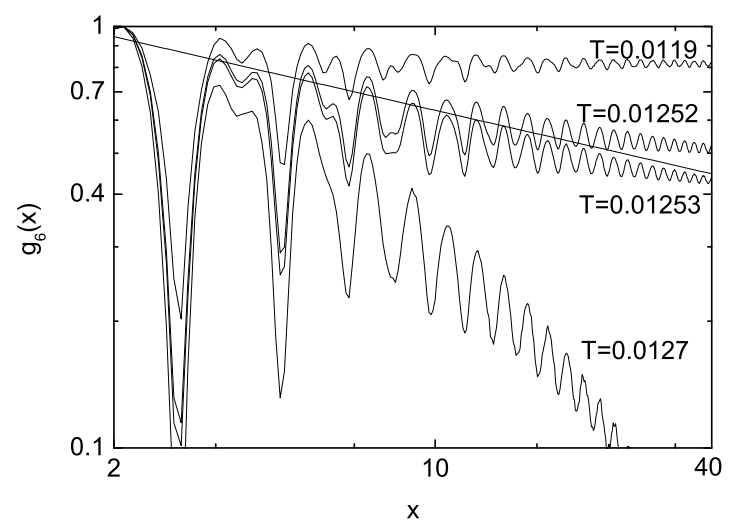

(a)

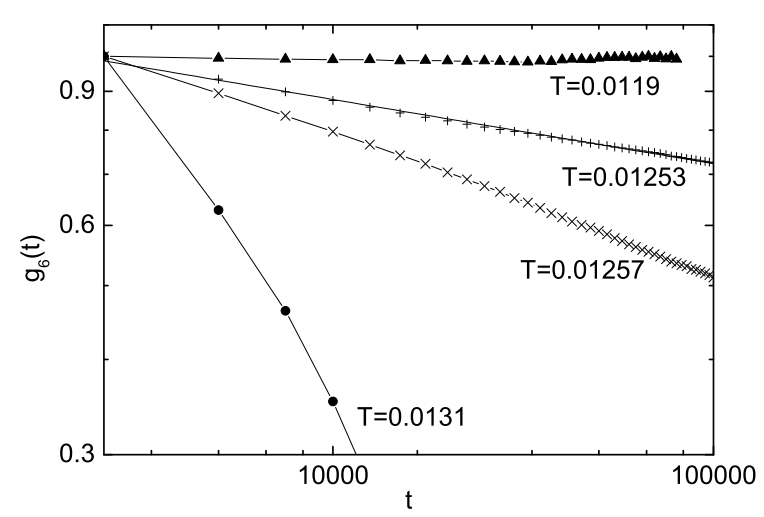

(b)

FIG. 3: (a) The spatial bond orientational correlation function $g_{6}(x)$ plotted vs. $x$ on a double decimal $\log$ scale. The temperature $T=0.0119$ is just before melting, $T=0.0127$ is typically in the liquid phase, and $T=0.01252$ and 0.01253 are in the hexatic phase. The straight line with a slope of $-1 / 4$ is a guide to the eyes. (b) The temporal bond orientational correlation function $g_{6}(t)$ plotted vs. $t$ on a double-log scale. The temperature $T=0.0119$ is just before melting, $T=0.0131$ is typically in the liquid phase, and $T=0.01253$ is in the hexatic phase. $g_{6}(t)$ at another $T=0.01257$, which is slight above the estimated $T_{i}$, is also shown. Lines fitted to the data are to guide the eyes. 


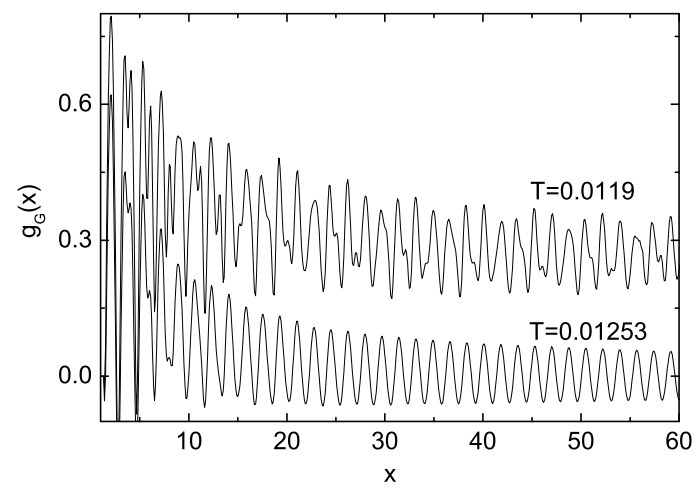

(a)

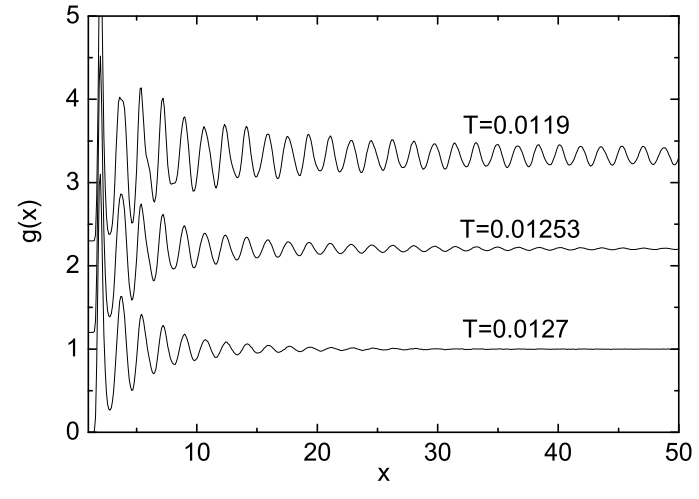

(b)

FIG. 4: (a) The positional correlation function $g_{G}(x)$ at $T=0.01253$ in the hexatic phase (lower curve) and 0.0119 in the solid phase (upper curve) plotted vs. $x$ on a linear scale. (b) The pair distribution function $g(x)$ plotted vs. $x$ on a linear scale. The upper two curve are shifted upward for clarity. The curves at $T=0.0119, T=0.01253$ and $T=0.0127$ show features in the solid, hexatic and liquid phases.

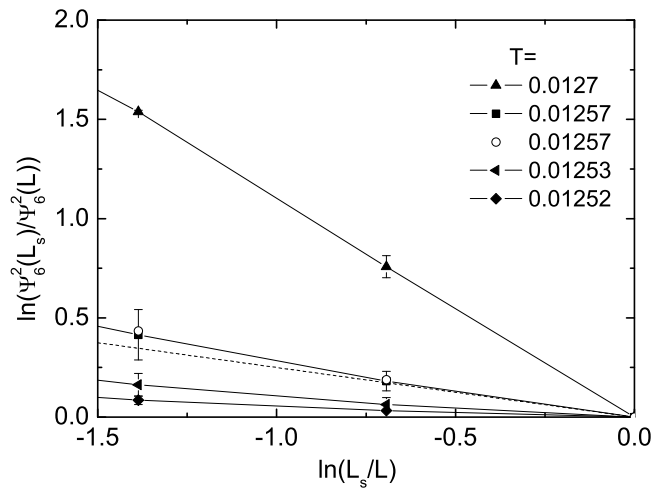

FIG. 5: The finite size scaling analysis of $\Psi_{6}^{2} . L=256$ or 512 is the bulk linear size and $L_{s}$ is the size of the subsystem. The dotted line with a slope of $-1 / 4$ is a guide to the eyes. Open circles are the results from independent simulations with periodic boundary conditions at $L=64,128,256$. 


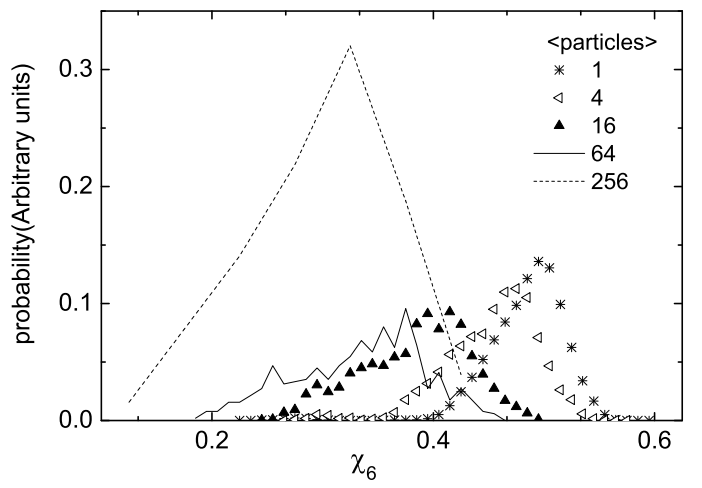

FIG. 6: The probability distribution of $\chi_{6}$ in the hexatic phase at $T=0.01252$. The symbols in the figure indicate the mean numbers of particles in different subsystem.

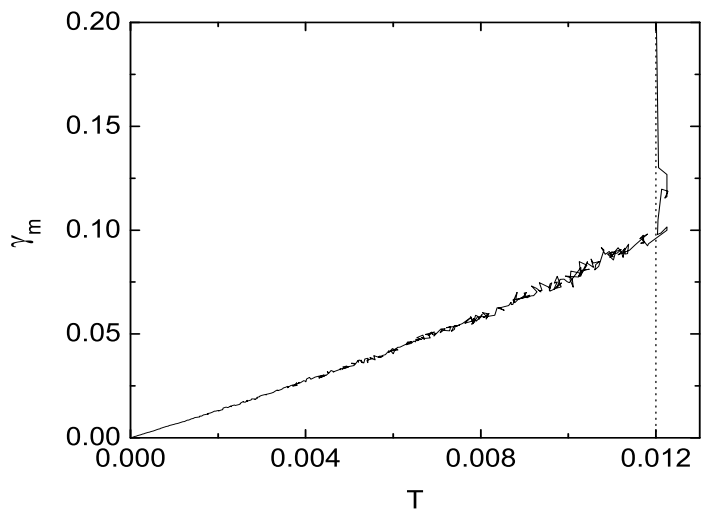

FIG. 7: The Lindemann parameter $\gamma_{m}$ vs. $T$. The critical temperature $T_{m}=0.0120$ is visualized by the vertical dotted line. 


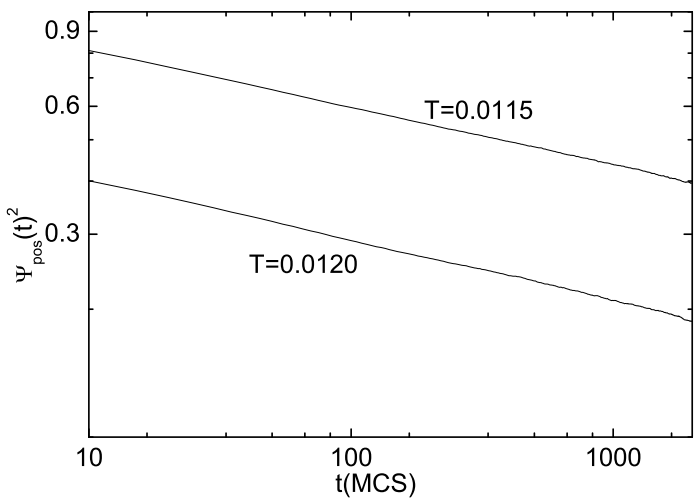

(a)

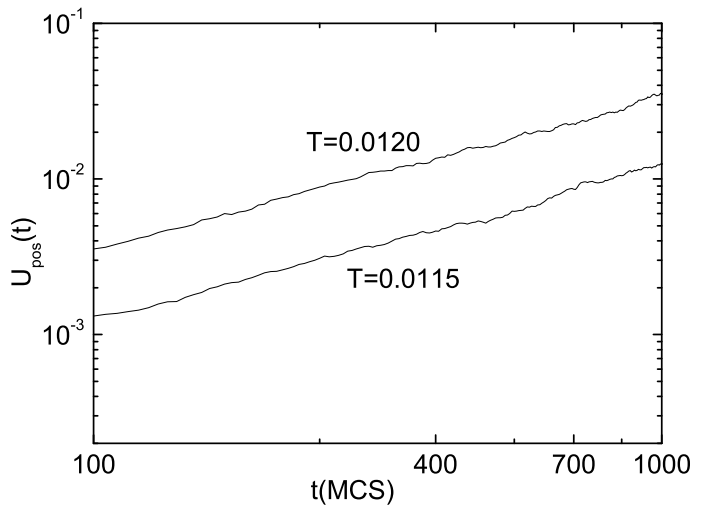

(b)

FIG. 8: (a) $\Psi_{\text {pos }}^{2}(t)$ plotted vs. $t$ on a double-log scale. The lower curve is shifted downward for clarity. (b) $U_{\text {pos }}(t)$ plotted vs. $t$ on a double-log scale. The upper curve is shifted upward for clarity. 